\title{
Genetic and Pharmacological Targeting of Transcriptional Repression in Resistance to Thyroid Hormone Alpha
}

\author{
Bernard Freudenthal, ${ }^{1, \star}$ Samiksha Shetty, ${ }^{1, *}$ Natalie C. Butterfield, ${ }^{1}$ John G. Logan, \\ Cho Rong Han, ${ }^{2}$ Xuguang Zhu, ${ }^{2}$ Inna Astapova, ${ }^{3}$ Anthony N. Hollenberg, ${ }^{4}$ \\ Sheue-Yann Cheng, ${ }^{2}$ J.H. Duncan Bassett, ${ }^{1}$ and Graham R. Williams ${ }^{1}$
}

Background: Thyroid hormones act in bone and cartilage via thyroid hormone receptor alpha (TR $\alpha)$. In the absence of triiodothyronine (T3), TR $\alpha$ interacts with co-repressors, including nuclear receptor co-repressor-1 (NCoR1), which recruit histone deacetylases (HDACs) and mediate transcriptional repression. Dominantnegative mutations of TR $\alpha$ cause resistance to thyroid hormone alpha (RTH $\alpha$; OMIM 614450), characterized by excessive repression of T3 target genes leading to delayed skeletal development, growth retardation, and bone dysplasia. Treatment with thyroxine has been of limited benefit, even in mildly affected individuals, and there is a need for new therapeutic strategies. It was hypothesized that (i) the skeletal manifestations of RTH $\alpha$ are mediated by the persistent TR $\alpha / \mathrm{NCoR} 1 / \mathrm{HDAC}$ repressor complex containing mutant $\mathrm{TR} \alpha$, and (ii) treatment with the HDAC inhibitor suberoylanilide hydroxamic acid (SAHA) would ameliorate these manifestations. Methods: The skeletal phenotypes of (i) Thral ${ }^{P V /+}$ mice, a well characterized model of RTH $\alpha$; (ii) $N c o r 1^{\Delta I D / \Delta I D}$ mice, which express an NCoR1 mutant that fails to interact with TR $\alpha$; and (iii) $T h r a 1^{P V /+} N \operatorname{cor} 1^{\Delta I D / \Delta I D}$ double-mutant adult mice were determined. Wild-type, Thral ${ }^{P V /+}, N \operatorname{corl} I^{\Delta I D / \Delta I D}$, and Thral ${ }^{P V /+} N \operatorname{corl} I^{\Delta I D / \Delta I D}$ double-mutant mice were also treated with SAHA to determine whether HDAC inhibition results in amelioration of skeletal abnormalities.

Results: Thral ${ }^{P V /+}$ mice had a severe skeletal dysplasia, characterized by short stature, abnormal bone morphology, and increased bone mineral content. Despite normal bone length, $N \operatorname{cor} 1^{\Delta I D / \Delta I D}$ mice displayed increased cortical bone mass, mineralization, and strength. Thral ${ }^{P V /+}{ } \operatorname{cor} 1^{\Delta I D / \Delta I D}$ double-mutant mice displayed only a small improvement of skeletal abnormalities compared to Thral ${ }^{P V /+}$ mice. Treatment with SAHA to inhibit histone deacetylation had no beneficial or detrimental effects on bone structure, mineralization, or strength in wild-type or mutant mice.

Conclusions: These studies indicate treatment with SAHA is unlikely to improve the skeletal manifestations of RTH $\alpha$. Nevertheless, the findings (i) confirm that TR $\alpha 1$ has a critical role in the regulation of skeletal development and adult bone mass, (ii) suggest a physiological role for alternative co-repressors that interact with TR in skeletal cells, and (iii) demonstrate a novel role for NCoR1 in the regulation of adult bone mass and strength.

Keywords: thyroid hormone receptor, RTH- $\alpha$, NCoR1, bone

\section{Introduction}

$\mathbf{T}$ RIIODOTHYRONINE (T3) ACTS mainly via thyroid hormone receptor alpha $(\mathrm{TR} \alpha)$ in bone and cartilage and regulates skeletal development, postnatal growth, and the maintenance of adult bone mass, mineralization, and strength
(1). Mutations affecting THRA encoding TR $\alpha$ cause resistance to thyroid hormone alpha (RTH $\alpha)(2-12)$, also classified as autosomal dominant non-goitrous congenital hypothyroidism type 6 (OMIM 614450).

THRA mutations result in a consistent pattern of thyroid function test abnormalities, comprising normal levels of

\footnotetext{
${ }^{1}$ Molecular Endocrinology Laboratory, Department of Medicine, Imperial College London, London, United Kingdom.

${ }^{2}$ Laboratory of Molecular Biology, National Cancer Institute, National Institutes of Health, Bethesda, Maryland.

${ }^{3}$ Endocrinology, Metabolism and Nutrition, Department of Medicine, Duke University School of Medicine, Durham, North Carolina.

${ }^{4}$ Joan and Sanford I. Weill Department of Medicine, Weill Cornell Medicine and New York Presbyterian/Weill Cornell Medical Center, New York, New York.

*These authors should be regarded as co-first authors.

(C) Bernard Freudenthal et al. 2019; Published by Mary Ann Liebert, Inc. This Open Access article is distributed under the terms of the Creative Commons License (http://creativecommons.org/licenses/by/4.0), which permits unrestricted use, distribution, and reproduction in any medium, provided the original work is properly cited.
} 
thyrotropin (TSH), a low or normal thyroxine (T4), and a high or normal T3 concentration. An elevated T3/T4 ratio is pathognomonic and present in all affected individuals (10). Individuals with $\mathrm{RTH} \alpha$ display a characteristic skeletal dysplasia consistent with impaired T3 action in bone and the skeletal consequences of severe congenital or juvenile hypothyroidism $(1,10)$. These include macrocephaly with patent fontanelles and cranial sutures, delayed tooth eruption, thickened calvarium with wormian sutures, delayed ossification and bone age, epiphyseal dysgenesis, and disproportionate short stature. Affected adults have cortical hyperostosis and increased bone mineral density (BMD). A phenotype-genotype correlation has been noted in the limited number of reported cases. Missense mutations are associated with a less severe phenotype than the profound dysplasia in individuals with truncation mutations that result in expression of potent dominant-negative mutant TR $\alpha$ proteins $(1,7,10)$. Consistent with this, the degree of dominant-negative activity of mutant $\mathrm{TR} \alpha$ also correlates with the clinical response to treatment with thyroid hormones $(6,10)$.

In $\operatorname{RTH} \alpha$, the mutant $\operatorname{TR} \alpha$ acts as a dominant-negative repressor of T3 target gene expression and an inhibitor of wild-type TR function (2). In the absence of T3, unliganded $\operatorname{TR} \alpha$ and $\operatorname{TR} \beta$ isoforms interact with transcriptional repressors, including nuclear receptor co-repressor-1 (NCoR1). This interaction leads to recruitment of histone deacetylase (HDAC) enzymes to a co-repressor complex, resulting in chromatin remodeling and inhibition of basal $\mathrm{T} 3$ target gene transcription (13). Binding of $\mathrm{T} 3$ causes a conformational change in the receptor and disrupts the interaction between TR and NCoR1. T3 binding thus promotes recruitment of nuclear receptor co-activators, such as steroid receptor coactivator 1, which possess histone acetyl transferase activity, leading to activation of T3 target gene expression (14). In $\mathrm{RTH} \alpha$, the mutant $\mathrm{TR} \alpha$ protein cannot release NCoR1 in response to T3, resulting in dominant repression of T3 target gene transcription because persistent HDAC-induced chromatin remodeling also prevents access for wild-type TRs to the transcriptional machinery. The disease phenotype in RTH $\alpha$ therefore reflects impaired T3 action in specific TR $\alpha$ dependent target tissues such as the skeleton, and its severity is directly related to the dominant-negative potency of the mutant receptor.

It was hypothesized that (i) the skeletal manifestations of $\mathrm{RTH} \alpha$ are mediated by the persistent TR $\alpha / \mathrm{NCOR} 1 / \mathrm{HDAC}$ repressor complex containing mutant $\mathrm{TR} \alpha$, and (ii) treatment with the HDAC inhibitor suberoylanilide hydroxamic acid (SAHA) would ameliorate the skeletal abnormalities.

To investigate these hypotheses, Thral ${ }^{P V /+}$ mice, which express a potent dominant negative mutant TR (TR $\alpha 1 \mathrm{PV})$ and recapitulate the $\mathrm{RTH} \alpha$ phenotype observed in individuals with similar THRA mutations, were studied (15-17). To determine the role of NCoR1 in the pathogenesis of the skeletal manifestations of $\mathrm{RTH} \alpha, \mathrm{Thral}^{P V /+}$ mice were crossed with Ncorl $1^{\Delta I D / \Delta I D}$ mice that express mutant NCoR1, which lacks the receptor interacting domains RID2 and RID3 required for TR binding but retains RID1 that interacts with other nuclear receptors $(13,18,19)$. Finally, wild-type, $T h r a 1^{P V / 4}, N_{c o r l}{ }^{\Delta I D / \Delta I D}$, and Thral ${ }^{P V / P}$ Norl $I^{\Delta I D / \Delta I D}$ double-mutant mice were treated with SAHA to determine whether HDAC inhibition results in amelioration of skeletal abnormalities in $\mathrm{RTH} \alpha$.

\section{Methods}

\section{Animals and treatment}

Animal studies were performed according to protocols approved by the National Cancer Institute Animal Care and Use Committee. Heterozygous $\mathrm{Thral}^{\mathrm{PV/T}}$ mice were generated in a mixed C57BL/6J and NIH Black Swiss genetic background and genotyped as described (17). Homozygous Ncorl $1^{\Delta I D / \Delta I D}$ mice were generated in a mixed C57BL/6 and 129S6 background as described (20). Thral ${ }^{P V /+}$ mice and Ncor $1^{\Delta I D / \Delta I D}$ mice were inter-crossed for several generations to produce Thral $^{P V /+} \mathrm{Ncorl}^{\Delta I D / \Delta I D}$ double mutants in a mixed C57BL/6J, NIH Black Swiss and 129S6 genetic background (21). Wild-type mice in a mixed C57BL/6J, NIH Black Swiss and 129S6 genetic background were used to ensure comparisons were made in as similar a genetic background as possible for all experiments ( $n=4-8$ per group).

SAHA (Selleck Chemicals, Houston, TX) or vehicle was prepared as described (22). A daily dose of $50 \mathrm{mg} / \mathrm{kg}$ body weight was administered by oral gavage for a two-month period starting at the age of six weeks until bones were harvested at 14 weeks of age.

\section{Faxitron digital $X$-ray microradiography}

Dissected femurs, humeri, and proximal caudal vertebrae $\mathrm{Ca} 6$ and $\mathrm{Ca} 7$ were imaged at $10 \mu \mathrm{m}$ resolution using a Faxitron MX20 (Qados; Cross Technologies plc, Sandhurst, United Kingdom). Bone lengths were determined after calibrating images with a digital micrometer using ImageJ (https://imagej.nih.gov/ij/). Bone mineral content (BMC) was determined relative to steel, aluminum, and polyester standards, and images were pseudocolored using a 16-color lookup table $(23,24)$.

\section{Micro computed tomography}

Femurs were imaged by micro computed tomography using a SCANCO $\mu \mathrm{CT} 50$ (SCANCO Medical AG, Bruttisellen, Switzerland) at $70 \mathrm{kV}, 200 \mu \mathrm{A}$, with a $0.5 \mathrm{~mm}$ aluminum filter and voxel resolutions of 5 and $10 \mu \mathrm{m}$ for trabecular and cortical bone, respectively. Images were reconstructed and analyzed using Scanco software. A $1 \mathrm{~mm}^{3}$ region of interest was selected $100 \mu \mathrm{m}$ from the growth plate, and trabecular bone volume as proportion of tissue volume (BV/TV), trabecular number (Tb.N), trabecular thickness (Tb.Th), and trabecular spacing (Tb.Sp) were determined $(23,25,26)$. Total cross-sectional area (Tt.Ar), cortical bone area (Ct.Ar), marrow or medullary area (Ma.Ar), and cortical area fraction (Ct.Ar/Tt.Ar) were also determined. A $1.5 \mathrm{~mm}$ region of interest, centered in the midshaft $56 \%$ along the length of the femur distal to the femoral head, was selected to determine cortical thickness (Ct.Th) and cortical BMD. Cortical porosity (Ct.Po), periosteal perimeter (Ps.Pm), and endocortical perimeter (Ec.Pm) were determined within a $0.5 \mathrm{~mm}$ region of interest centered in the midshaft $56 \%$ along the length of the femur distal to the femoral head from images with a voxel resolution of $1 \mu \mathrm{m}$.

\section{Biomechanical testing}

Destructive three-point bend tests were performed on $70 \%$ ethanol-fixed humeri to determine bone strength using an 
Instron 5543 load frame and $100 \mathrm{~N}$ load cell (Instron Limited, High Wycombe, United Kingdom). Humeri were positioned horizontally on custom supports, and load was applied perpendicular to the mid-diaphysis at a constant rate of displacement of $0.03 \mathrm{~mm} / \mathrm{s}$ until fracture. Yield load, maximum load, fracture load, and stiffness were determined from load displacement curves $(23,27)$.

\section{Statistical analysis}

Data were normally distributed and analyzed by analysis of variance and Tukey's post hoc test or unpaired two-tailed Student's $t$-test. $p$-Values $<0.05$ were considered statistically significant. Cumulative and relative frequency distributions of BMC were compared using the Kolmogorov-Smirnov test $(23,24)$.

\section{Results}

\section{Expression of NCOR1DID ameliorates bone structural} defects in Thra $1^{\mathrm{PV} /+}$ mice and increases bone strength

In 14-week-old adult Thral ${ }^{P V /+}$ mice, the lengths of the femurs, humeri, and vertebrae were decreased by $12 \%, 16 \%$, and $30 \%$, respectively, and BMC was increased $(p<0.001)$. The changes in bone length and BMC were accompanied by morphological abnormalities that included dysmorphic epiphyses with misshapen joints and splayed metaphyses with defective inwasting (Fig. 1A). Similar dysplastic features were evident in Thral ${ }^{P V /+} N \operatorname{corl} l^{\Delta I D / \Delta I D}$ double-mutant mice, in which bone lengths were also decreased and BMC increased compared to wild-type mice. Nevertheless, reductions in the lengths of the humerus and vertebrae in Thral ${ }^{P V /+}{ }^{N}$ corl $1^{\Delta I D / \Delta I D}$ double mutants were less than those observed in Thral $^{P V /+}$ mice (Fig. 1A). Femurs from Thral ${ }^{P V /+}$ mice had reduced $\mathrm{Ct}$.Th and increased Ct.Po but no difference in BMD compared to wild-type mice (Fig. 1B). These parameters were accompanied by increased Tt.Ar, Ma.Ar, Ps.Pm, and Ec.Pm, no difference in Ct.Ar, and a decrease in Ct.Ar/Tt.Ar (Supplementary Fig. S1), indicating an overall decrease in cortical bone thickness together with increased porosity but normal BMD. Thral ${ }^{P V /+}$ mice also had high trabecular bone mass, as evidenced by a threefold increase in $\mathrm{BV} / \mathrm{TV}$, a twofold increase in Tb.N, and a twofold reduction in Tb.Sp (Fig. 1C). Thral ${ }^{P V /+}$ corl ${ }^{\Delta I D / \Delta I D}$ double-mutant mice had a small increase in BMD and increased Ct.Po (Fig. 1B) but high trabecular bone mass, with a 2.5 -fold increase in BV/TV, a $50 \%$ increase in Tb.N, a $10 \%$ increase in $\mathrm{Tb} . \mathrm{Th}$, and a $62.5 \%$ reduction in $\mathrm{Tb} . \mathrm{Sp}$ (Fig. 1C). The changes in cortical and trabecular bone parameters were not as large in $T h r a l^{P V /+} N \operatorname{corl} l^{\Delta I D / \Delta I D}$ double mutants compared to Thral $^{P V /+}$ mice (Fig. $1 \mathrm{~B}$ and $\mathrm{C}$ ), other than an increase in cortical area (Supplementary Fig. S1). The structural abnormalities in $\mathrm{Thral}^{P V /+}$ mice resulted in humeri that were weak, with an $18 \%$ decrease in yield load and $16 \%$ decrease in maximum load, whereas humeri from Thral ${ }^{P V /+} N \operatorname{cor} l^{\Delta I D / \Delta I D}$ double-mutant mice were stronger, with a $16 \%$ increase in maximum load and $24 \%$ increase in fracture load compared to wild-type mice (Fig. 1D).

Overall, expression of NCoR1 $\Delta$ ID in double-mutant mice ameliorated the structural abnormalities evident in bones from Thral $^{P V /+}$ mice and ultimately resulted in increased adult bone strength, consistent with the increased cortical area observed in Thral ${ }^{P V /+}$ Norl $1^{\Delta I D / \Delta I D}$ double mutants.

\section{Expression of NCoR1DID increases bone mass, mineralization, and strength}

In 14-week-old adult $N \operatorname{cor} 1^{\Delta I D / \Delta I D}$ mice, skeletal morphology and the lengths of the femurs, humeri, and vertebrae were similar to wild-type mice, but femurs had increased BMC (Fig. 2A) that resulted from a combination of an $18 \%$ increase in $\mathrm{Ct}$.Th, a small increase in cortical BMD, and an increase in Ct.Po (Fig. 2B). There were no differences in trabecular bone parameters (Fig. 2C). The increased cortical bone in Ncorl $1^{\triangle I D / \Delta I D}$ mice (Fig. 2B and Supplementary Fig. S2) resulted in increased bone strength compared to wild-type mice, as evidenced by a $30 \%$ increase in maximum load, a $35 \%$ increased fracture load, and a $20 \%$ increase in stiffness (Fig. 2D).

Overall, expression of NCoR $1 \Delta \mathrm{ID}$ results in increased cortical bone mass, mineralization, and strength in adult Nor $1^{\triangle I D / \Delta I D}$ mice despite an increase in Ct.Po.

\section{Treatment with SAHA has no effect on bone mass, mineralization, or strength}

Two months of treatment with the histone deacetylase inhibitor SAHA between 6 and 14 weeks of age had no effect on bone structure, mineralization, or strength in either wild-type or Thral ${ }^{P V /+}$ mice, other than a small decrease in femur BMC in Thral ${ }^{P V /+}$ mice (Fig. 3 and Supplementary Fig. S3). Similarly, treatment of Ncorl ${ }^{\Delta I D / \Delta I D}$ and Thral ${ }^{P V /+}$ Nor $1^{\Delta I D / \Delta I D}$ doublemutant mice with SAHA had no effect on any parameter, other than a small reduction in the length of the humerus present in $T h r a l^{P V /+} N \operatorname{corl} l^{\Delta I D / \Delta I D}$ double-mutant mice treated with SAHA (Fig. 4 and Supplementary Fig. S4).

Overall, treatment with SAHA had no beneficial or detrimental effect on bone structure, mineralization, or strength in wild-type or mutant mice.

\section{Discussion}

$\mathrm{RTH} \alpha$ results in delayed skeletal and dental development, growth retardation, and skeletal dysplasia, as well as additional non-skeletal manifestations primarily affecting the central nervous, gastro-intestinal, and metabolic systems (10). To date, 11 individuals have received treatment with T4, but other therapeutic possibilities have not been investigated $(2-5,8,9,12)$. Children with less severe frameshift THRA mutations have derived only limited benefit after treatment with T4, particularly with respect to growth $(2,8,12)$, but no skeletal responses have been seen in individuals with deleterious frame-shift truncation mutations $(6,9,28)$. Thus, there is a need to investigate new therapeutic strategies for treatment of the debilitating skeletal manifestations of RTH $\alpha$. Here, two complementary approaches to disrupt the detrimental dominant-negative activity of mutant $\mathrm{TR} \alpha$ in the Thral ${ }^{P V /+}$ mouse model of $\mathrm{RTH} \alpha$ were investigated.

First, T3 action in bone is mediated by the canonical genomic actions of $\operatorname{TR} \alpha 1(1,26)$, while the skeletal dysplasia in $\mathrm{RTH} \alpha$ results from (i) impaired tissue T3 responsiveness in bone and cartilage despite a moderately increased circulating $\mathrm{T} 3$ concentration and (ii) the dominant-negative repressor activity of mutant $\mathrm{TR} \alpha 1$ that results from its inability to 


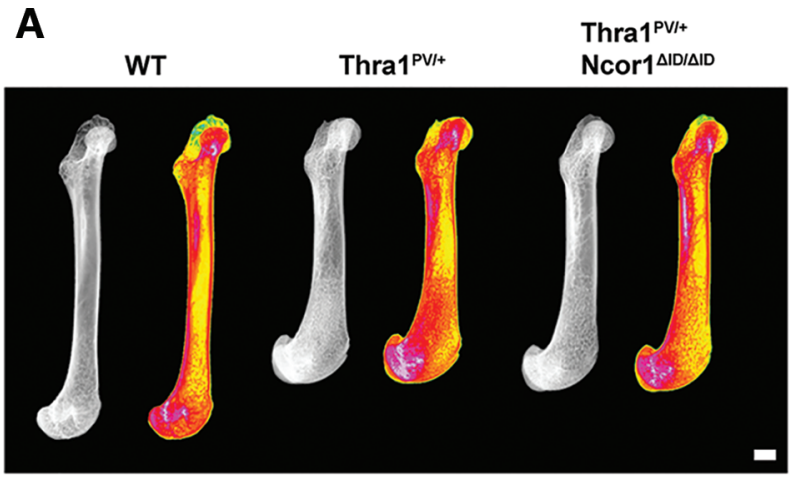

WT $\square \quad$ Thra1 $^{\mathrm{PVI+}} \square \quad$ Thra1 $^{\mathrm{PV} /+}$ Ncor1 ${ }^{\Delta I D / \Delta I D} \square$
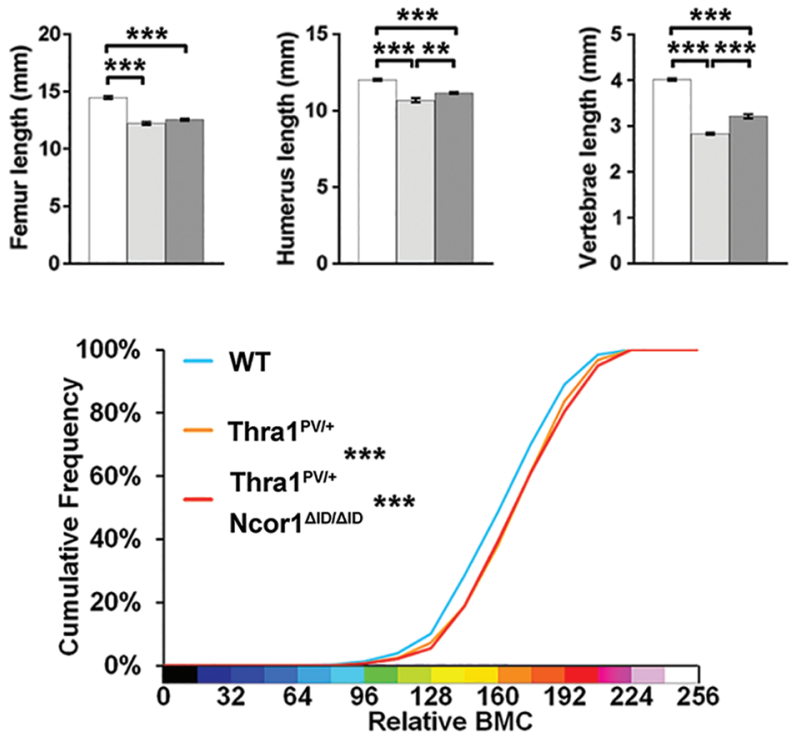

D

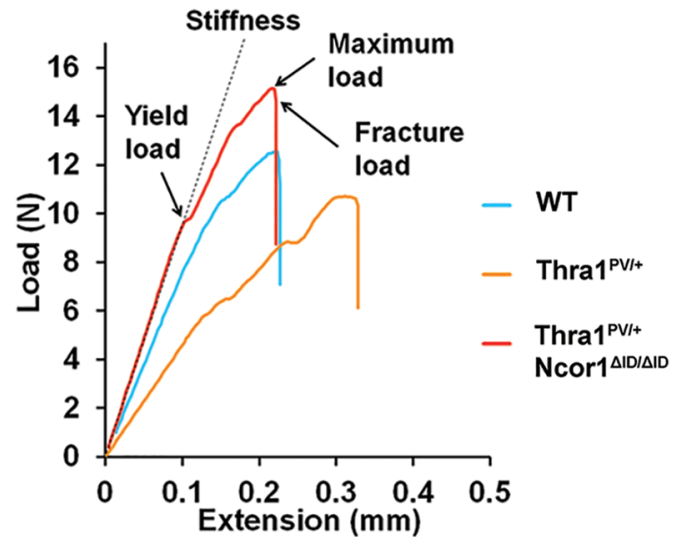

B
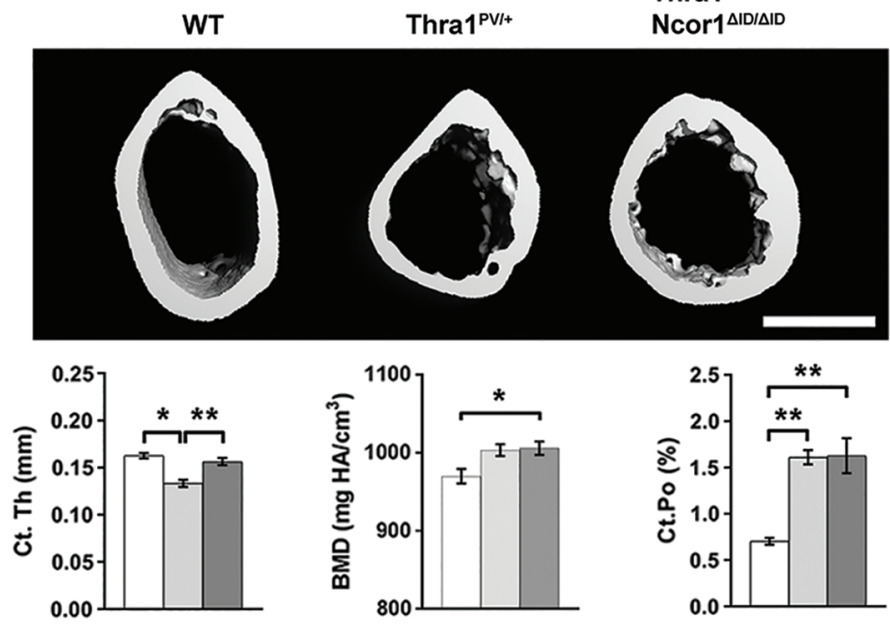

Thra ${ }^{\mathrm{PV} /+}$

C wT

Thra1 $^{\mathrm{PV} /+}$
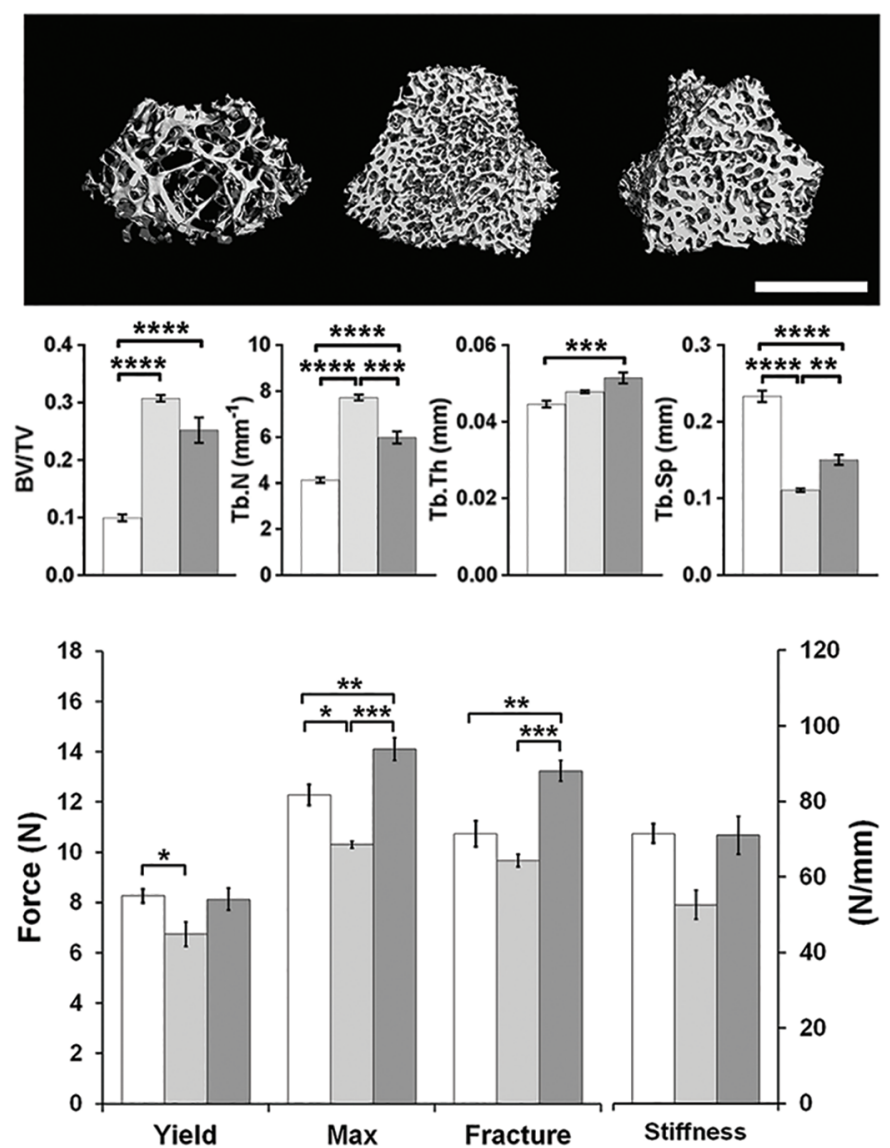

FIG. 1. Disruption of the interaction between nuclear receptor co-repressor-1 (NcoR1) and thyroid hormone receptor alpha $(\mathrm{TR} \alpha)$ ameliorates the skeletal phenotype in $\mathrm{Thral}^{P V /+}$ mice. (A) X-ray microradiography images of femurs from male wildtype (WT), Thral ${ }^{P V /+}$, and Thral ${ }^{P V /} N C O R 1^{\Delta I D / \Delta I D}$ mice at 14 weeks of age. Gray-scale images and pseudocolored images in which low bone mineral content (BMC) is indicated in green and high BMC in pink. Cumulative frequency histogram of relative BMC $(n=5-8$ per group). *** $p<0.001$ vs. WT; Kolmogorov-Smirnov test. Graphs show lengths of femurs, humeri, and vertebrae. Data are shown as the mean \pm standard error of the mean (SEM; $n=4-8$ per group). ${ }^{* *} p<0.01$ and $* * * p<0.001$; analysis of variance (ANOVA) followed by Tukey's post hoc test. (B) Micro computed tomography (micro-CT) images of middiaphysis cortical bone. Graphs showing cortical thickness (Ct.Th), cortical bone mineral density (BMD), and cortical porosity (Ct.Po). Data are shown as the mean \pm SEM $(n=5-8$ per group apart from Ct.Po, $n=3) .{ }^{*} p<0.05$ and ${ }^{*} p<0.01$; ANOVA followed by Tukey's post hoc test. (C) Micro-CT images of distal femur trabecular bone. Graphs showing trabecular bone volume/tissue volume (BV/TV), trabecular number (Tb.N), trabecular thickness (Tb.Th), and trabecular spacing (Tb.Sp). Data are shown as the mean $\pm \operatorname{SEM}\left(n=5-8\right.$ per group). ${ }^{* *} p<0.01, * * * p<0.001$, and $* * * * p<0.0001$; ANOVA followed by Tukey's post hoc test. (D) Representative load displacement curves for humerus three-point bend testing. Yield load, maximum load, fracture load, and stiffness. Data are the mean \pm SEM ( $n=5-8$ per group). ${ }^{*} p<0.05,{ }^{* *} p<0.01$, and ${ }^{* * *} p<0.001$; ANOVA followed by Tukey's post hoc test. Scale bars in $(\mathbf{A}),(\mathbf{B})$ and $(\mathbf{C})=1 \mathrm{~mm}$. Data from the same group of untreated WT mice are included in Figures 1-4 to facilitate comparison across groups. Color images are available online. 
A
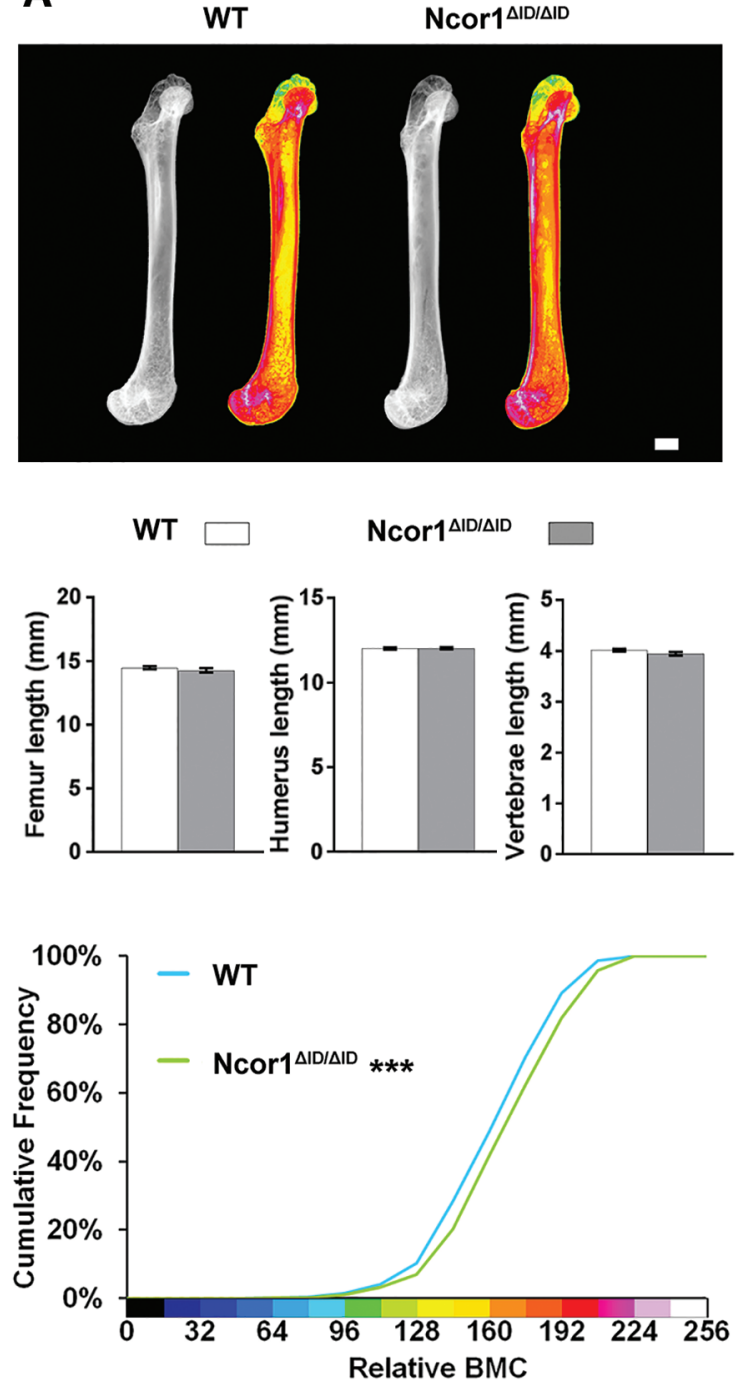

D

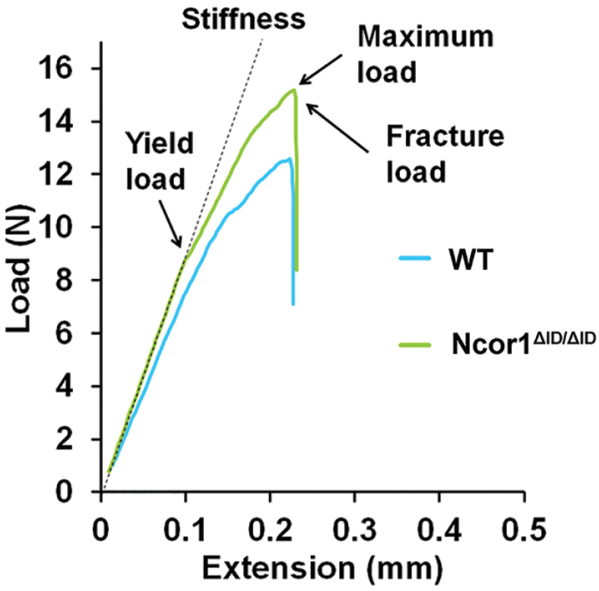

B

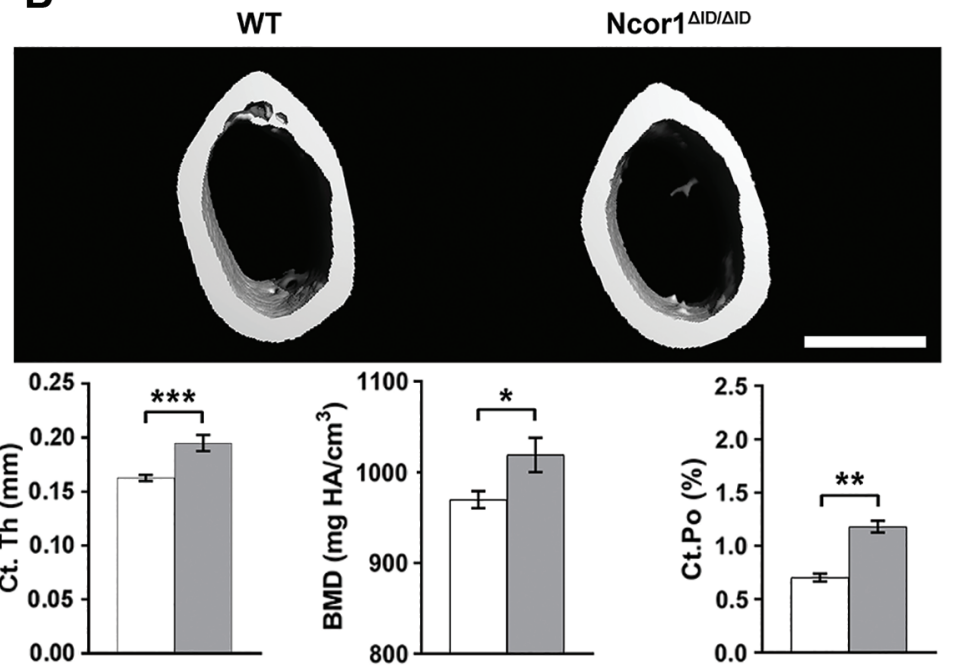

C

WT

Ncor1 ${ }^{\Delta I D / \Delta I D}$
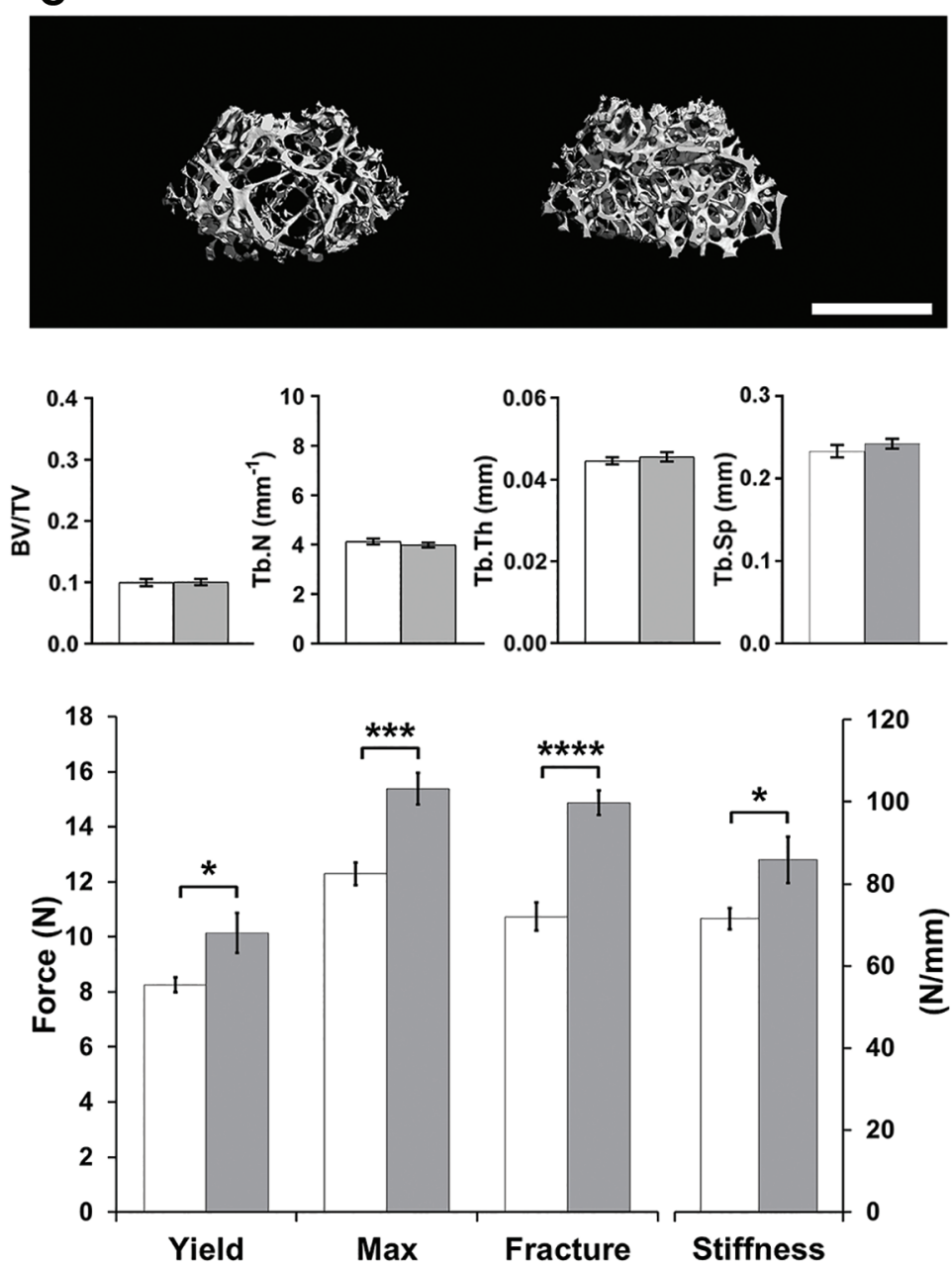

FIG. 2. Disruption of the interaction between NcoR1 and TR $\alpha$ increases bone mass, mineralization, and strength in WT mice. (A) X-ray microradiography images of femurs from male WT and $N C o R 1^{\Delta I D / \Delta I D}$ mice at 14 weeks of age. Gray-scale images and pseudocolored images in which low BMC is indicated in green and high BMC in pink. Cumulative frequency histogram of relative BMC ( $n=6-8$ per group). *** $p<0.001$ vs. WT; Kolmogorov-Smirnov test. Graphs show lengths of femurs, humeri, and vertebrae. Data are shown as the mean $\pm \operatorname{SEM}(n=6-8$ per group); Student's $t$-test. (B) Micro-CT images of mid-diaphysis cortical bone. Graphs showing Ct.Th, cortical BMD, and Ct.Po. Data are shown as the mean \pm SEM $(n=6-8$ per group apart from Ct.Po, $n=3)$. * $p<0.05$, $* * p<0.01$, and ***p $<0.001$; Student's $t$-test. (C) Micro-CT images of distal femur trabecular bone. Graphs showing BV/TV, Tb.N, Tb.Th, and Tb.Sp. Data are shown as the mean $\pm \operatorname{SEM}(n=6-8$ per group). (D) Representative load displacement curves for humerus three-point bend testing. Yield load, maximum load, fracture load, and stiffness. Data are the mean \pm SEM ( $n=6-8$ per group). $* p<0.05$, *** $p<0.001$, and $* * * * p<0.0001$ vs. WT; Student's $t$-test. Scale bars in $(\mathbf{A}),(\mathbf{B})$ and $(\mathbf{C})=1 \mathrm{~mm}$. Data from the same group of untreated WT mice are included in Figures 1-4 to facilitate comparison across groups. Color images are available online. 


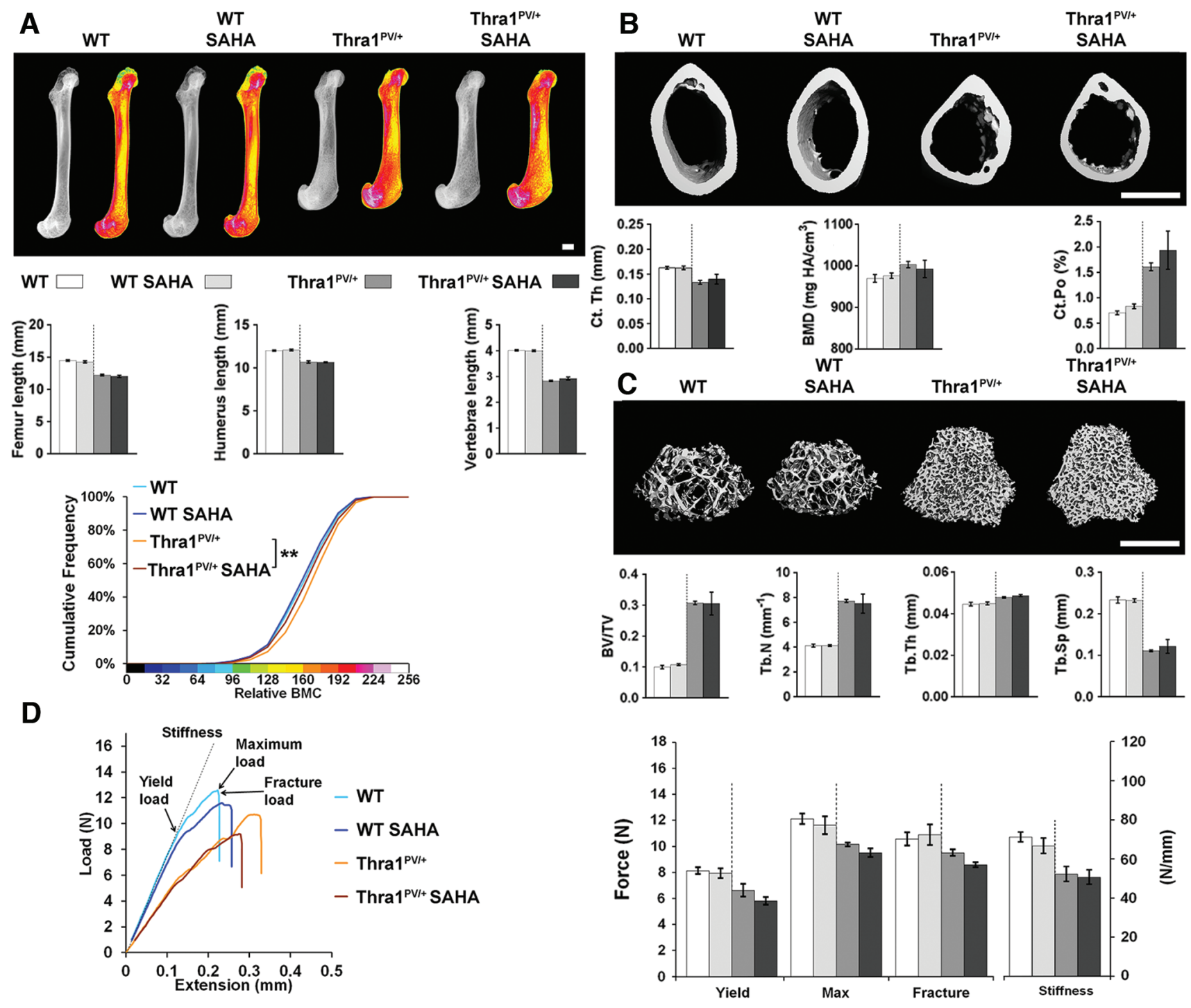

FIG. 3. Treatment with suberoylanilide hydroxamic acid (SAHA) has no effect on bone mass, mineralization, or strength in WT or Thral ${ }^{P V /+}$ mice. (A) X-ray microradiography images of femurs from male WT, SAHA-treated WT (WT SAHA), Thral $^{P V /+}$, and SAHA-treated Thral ${ }^{P V /+}$ (Thral ${ }^{P V /+}$ SAHA) mice at 14 weeks of age. Gray-scale images and pseudocolored images in which low BMC is indicated in green and high BMC in pink. Cumulative frequency histogram of relative BMC ( $n=5-8$ per group). $* * p<0.01$ SAHA treated vs. untreated; Kolmogorov-Smirnov test. Graphs show lengths of femurs, humeri, and vertebrae. Data are shown as the mean $\pm \operatorname{SEM}(n=5-8$ per group), treated vs. untreated; Student's $t$-test. (B) Micro-CT images of mid-diaphysis cortical bone. Graphs showing Ct.Th, cortical BMD, and Ct.Po. Data are shown as the mean \pm SEM $(n=5-8$ per group apart from Ct.Po, $n=3)$. (C) Micro-CT images of distal femur trabecular bone. Graphs showing BV/TV, Tb.N, Tb.Th, and Tb.Sp. Data are shown as the mean \pm SEM ( $n=5-8$ per group). (D) Representative load displacement curves for humerus three-point bend testing. Yield load, maximum load, fracture load, and stiffness. Data are the mean \pm standard error of the mean $(n=5-8$ per group); treated vs. untreated. Scale bars in $(\mathbf{A}),(\mathbf{B})$ and $(\mathbf{C})=1 \mathrm{~mm}$. Data from the same group of untreated WT mice are included in Figures 1-4 to facilitate comparison across groups. Color images are available online.

release co-repressor in the presence of T3 (13). Normally, NCoR1 interacts with TR via two interaction domains and is the main co-repressor that recruits HDACs to mediate transcriptional repression in the absence of $\mathrm{T} 3$. The functional interaction between TR $\alpha 1$ and NCoR1 can be targeted elegantly in mice by the $N \operatorname{corl}^{\Delta I D}$ mutation $(13,18,19)$, which prevents association between NCoR1 and unliganded TR and thus relieves NCoR1-dependent transcriptional repression $(21,29-31)$.

It was previously shown that similar to patients with $\mathrm{RTH} \alpha$, Thral $^{\mathrm{PV/+}}$ mice have an abnormal hypothalamic- pituitary-thyroid (HPT) axis, with a moderately increased T3 concentration, normal T4, slightly elevated TSH, and an increased size of the thyroid gland (21). Ncorl $1^{\triangle I D / \Delta I D}$ mice by contrast have decreased $\mathrm{T} 3$ and $\mathrm{T} 4$ levels but a normal TSH and normal sized thyroid gland. Thral ${ }^{P V /+}$ Ncorl ${ }^{\Delta I D / \Delta I D}$ double-mutant mice have a normal T3, decreased T4, normal $\mathrm{TSH}$, and a slightly enlarged thyroid gland. Thus, coexpression of NCoR1 IID in Thral ${ }^{P V /+} \operatorname{corr}^{\Delta I D / \Delta I D}$ double mutants ameliorates dysregulation of the HPT axis and normalizes the increased T3 and TSH levels that are caused by dominant-negative actions of TR $\alpha 1 \mathrm{PV}$ in $\mathrm{Thral}^{\mathrm{PV/+}}$ mice 


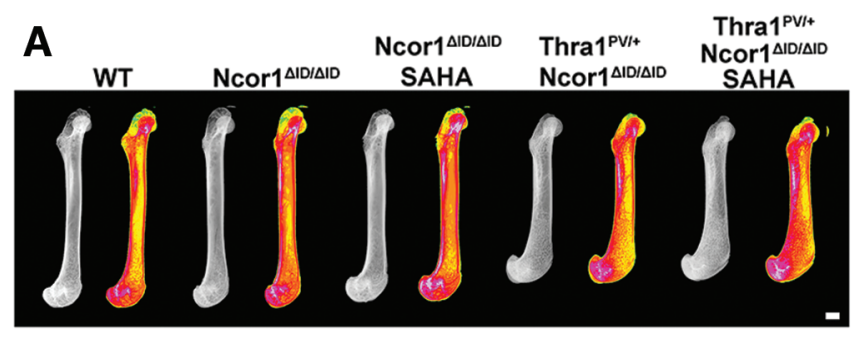

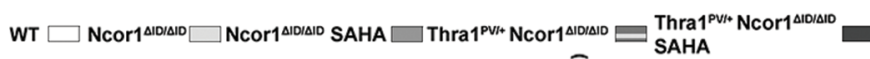
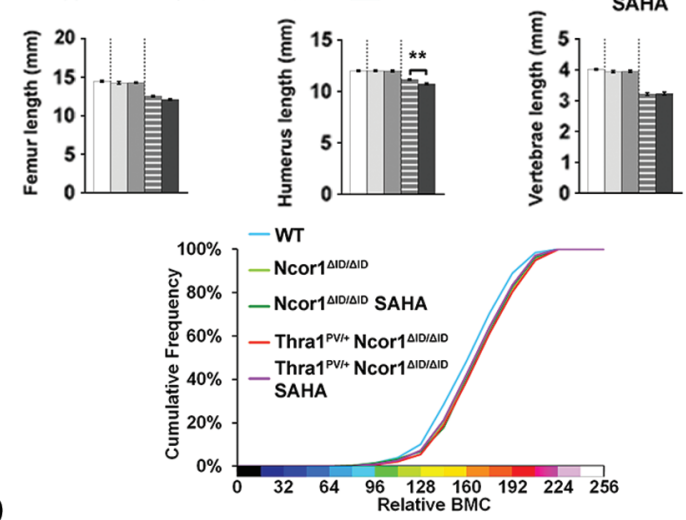

D
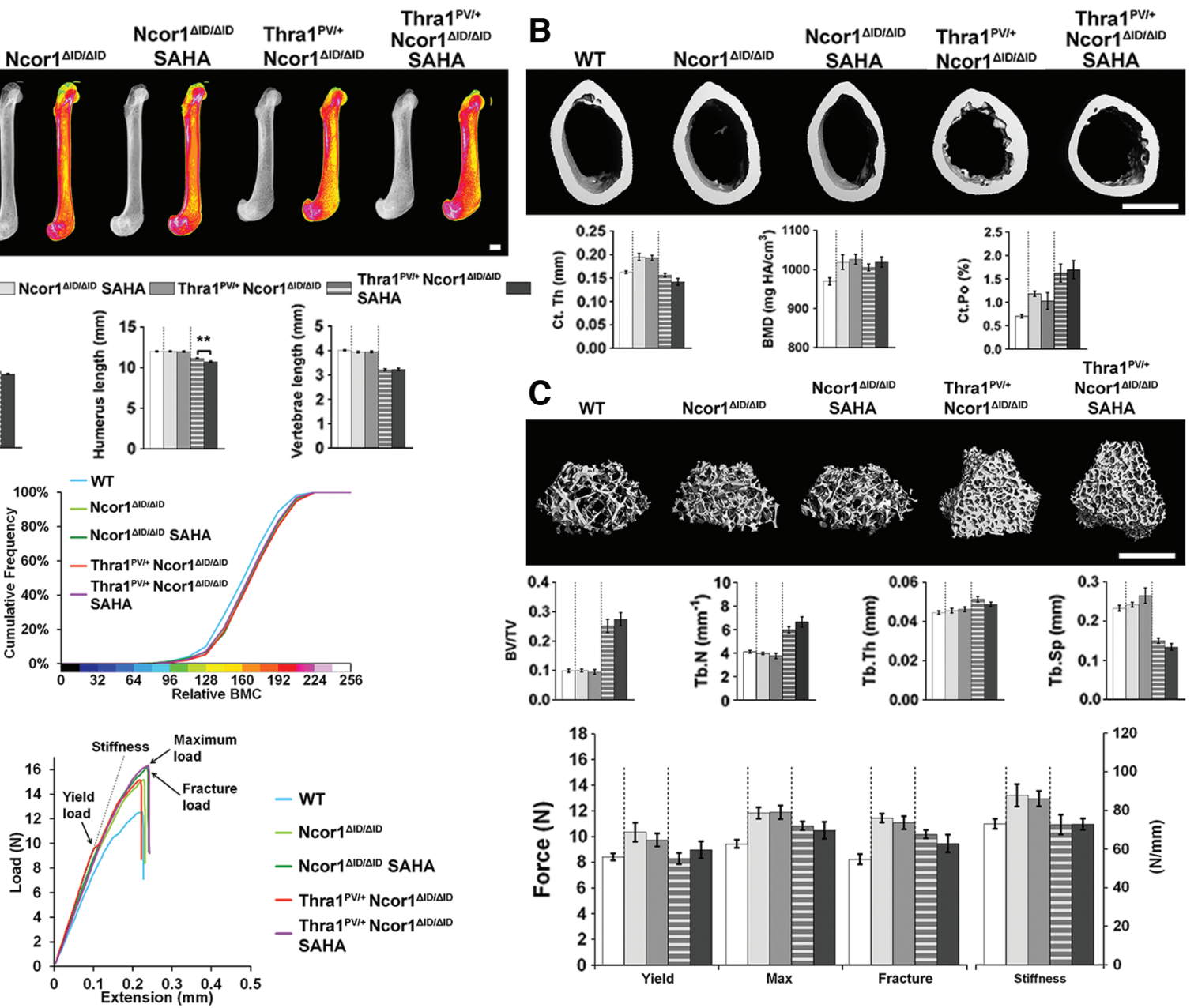

FIG. 4. Treatment with SAHA has no effect on bone mass, mineralization, or strength in $N C o R 1^{\Delta I D / \Delta I D}$ or $T h r a 1^{P V /+} N C o R 1^{\Delta I D / \Delta I D}$ mice. (A) X-ray microradiography images of femurs from male WT, $N C o R 1^{\Delta I D / \Delta I D}$, SAHA-treated $N C O R 1^{\Delta I D / \Delta I D}\left(N C o R 1^{\Delta I D / \Delta I D}\right.$ SAHA), Thral ${ }^{P V /+} N C o R 1^{\Delta I D / \Delta I D}$, and SAHA-treated Thral ${ }^{P V /+}{ }^{N C o R 1^{\Delta I D / \Delta I D}}$ (Thral ${ }^{P V /+} N C o R 1^{\Delta I D / \Delta I D}$ SAHA) mice at 14 weeks of age. Gray-scale images and pseudocolored images in which low BMC is indicated in green and high BMC in pink. Cumulative frequency histogram of relative BMC ( $n=6-8$ per group). SAHA treated vs. untreated; Kolmogorov-Smirnov test. Graphs show femur, humerus and vertebral lengths. Data are shown as the mean $\pm \operatorname{SEM}(n=6-8$ per group); treated vs. untreated. ${ }^{* *} p<0.01$; Student's $t$-test. (B) Micro-CT images of mid-diaphysis cortical bone. Graphs showing Ct.Th, cortical BMD, and Ct.Po. Data are shown as the mean $\pm \operatorname{SEM}(n=6-8$ per group apart from Ct.Po, $n=3)$. (C) Micro-CT images of distal femur trabecular bone. Graphs showing BV/TV, Tb.N, Tb.Th, and Tb.Sp. Data are shown as the mean $\pm \operatorname{SEM}(n=6-8$ per group). (D) Representative load displacement curves for humerus three-point bend testing. Yield load, maximum load, fracture load, and stiffness. Data are the mean $\pm \operatorname{SEM}(n=6-8$ per group); treated vs. untreated. Scale bars in $(\mathbf{A}),(\mathbf{B})$ and $(\mathbf{C})=1 \mathrm{~mm}$. Data from the same group of untreated WT mice are included in Figures 1-4 to facilitate comparison across groups. Color images are available online.

(21). By contrast, treatment of Thral ${ }^{P V /+}, N \operatorname{corl} 1^{\Delta I D / \Delta I D}$, and Thral ${ }^{P V /+} N$ corl ${ }^{\Delta I D / \Delta I D}$ double-mutant mice with SAHA does not affect circulating T3, T4, or TSH concentrations (22).

Disruption of the interaction between TR $\alpha 1$ and NCoR1 in $N$ corl $1^{\triangle I D / \Delta I D}$ mice resulted in increased cortical bone mass, mineralization, and strength but did not affect linear growth or trabecular bone parameters (Fig. 2). These findings demonstrate a new and important physiological and homeostatic function of NCoR1 affecting the skeleton. Thus, a lack of interaction between NCoR1 and TR isoforms has an anabolic impact on cortical bone to improve bone strength, whereas global interactions between NCoR1 and TRs limit cortical bone accumulation and decrease bone strength. They also reveal that $\mathrm{NCoR} 1$ is dispensable during endochondral bone formation and postnatal growth. Consistent with this new role for NCoR1 in bone, disruption of the interaction between dominant-negative $\mathrm{TR} \alpha 1^{\mathrm{PV}}$ and NCoR1 in Thral $^{P V / 4}$ Ncorl $^{\Delta I D / \Delta I D}$ double-mutant mice also increased cortical bone mass and strength but had only limited effects on skeletal morphology, linear growth, and trabecular bone mass (Fig. 1). Importantly, comparison of $\mathrm{Ncorl}^{\Delta I D / \Delta I D}$ mice (Fig. 2) with Thral ${ }^{P V /+} N \operatorname{cor} 1^{\Delta I D / \Delta I D}$ double-mutant mice (Fig. 1) reveals that $T h r a l^{P V /+} N \operatorname{cor} I^{\Delta I D / \Delta I D}$ mice have decreased bone strength parameters. This effect of TR $\alpha 1^{\mathrm{PV}}$ to worsen the phenotype of $N \operatorname{corl}^{\Delta I D / \Delta I D}$ mice is independent of NCoR 1 function and thus provides evidence of a role for additional co-repressors that may interact with $\mathrm{TR} \alpha$ in skeletal cells. 
Our second approach was to investigate pharmacological inhibition of excessive HDAC activity in RTH $\alpha$. SAHA chelates zinc ions required for histone deacetylase activity and transcriptional repression, thus resulting in enzyme inhibition, increased histone acetylation, and transcriptional activation (32). SAHA has received approval from the U.S. Food and Drug Administration for the treatment of certain types of cancer and thus could be repurposed for other disease applications if effective. Accordingly, SAHA has been proposed as a potential drug to relieve the detrimental consequences of transcriptional repression in $\mathrm{RTH} \alpha$, and has been shown to ameliorate impaired adipogenesis in Thral ${ }^{P V /+}$ mice at the same dose used in the current study (22). Despite this, it was found that pharmacological inhibition of excessive HDAC activity in $T h r a l^{P V /+}$ and $T h r a l^{P V /+} N$ cor $l^{\Delta I D / \Delta I D}$ double-mutant mice had no beneficial effect on linear growth or adult bone structure and strength (Figs. 3 and 4). This lack of skeletal response to SAHA may be due to (i) its short halflife following once-daily oral administration in mice (33), (ii) commencement of treatment at six weeks of age after the period of maximum linear growth velocity (34), and (iii) its potentially limited bioavailability in bone and cartilage. Overall, the current data suggest that SAHA is unlikely to have therapeutic benefit in the treatment of skeletal manifestations in RTH $\alpha$.

In summary, the current studies demonstrate that TR $\alpha 1$ exerts major regulatory effects on linear growth and adult bone turnover that are independent of NCoR1. Overall, the findings suggest that the skeletal manifestations of RTH $\alpha$ are not mediated by persistent interactions between mutant $\mathrm{TR} \alpha$ with NCoR1 and HDAC. The limited improvement of skeletal abnormalities in $\mathrm{RTH} \alpha$ following disruption of the interaction between the dominant-negative TR and NCoR1 further suggests an important role for alternative co-repressors that interact with TR in skeletal cells such as silencing mediator of retinoid and thyroid hormone receptors (13).

Despite this, these studies identify a novel physiological role for NCoR1 in association with TRs to (i) optimize bone strength and (ii) limit excessive accumulation of cortical bone.

\section{Acknowledgments}

B.F. was supported by a Medical Research Council Clinical Research Training Fellowship (MR/P018718/1). A.N.H. received funding from a National Institutes of Health (NIH) National Institute of Diabetes and Digestive and Kidney Diseases R01 Grant (DK056123). S.-Y.C. is funded by NIH Intramural Research. J.H.D.B. and G.R.W. are funded by a Wellcome Trust Joint Investigator Award (110141/Z/15/Z and 110140/Z/15/Z).

\section{Author Disclosure Statement}

No competing financial interests exist for any of the authors.

\section{Supplementary Material}

Supplementary Figure S1

Supplementary Figure S2

Supplementary Figure S3

Supplementary Figure S4

\section{References}

1. Bassett JH, Williams GR 2016 Role of thyroid hormones in skeletal development and bone maintenance. Endocr Rev 37:135-187.

2. Bochukova E, Schoenmakers N, Agostini M, Schoenmakers E, Rajanayagam O, Keogh JM, Henning E, Reinemund J, Gevers E, Sarri M, Downes K, Offiah A, Albanese A, Halsall D, Schwabe JW, Bain M, Lindley K, Muntoni F, Vargha-Khadem F, Dattani M, Farooqi IS, Gurnell M, Chatterjee K 2012 A mutation in the thyroid hormone receptor alpha gene. N Engl J Med 366:243-249.

3. Espiard S, Savagner F, Flamant F, Vlaeminck-Guillem V, Guyot R, Munier M, d'Herbomez M, Bourguet W, Pinto G, Rose C, Rodien P, Wemeau JL 2015 A novel mutation in THRA gene associated with an atypical phenotype of resistance to thyroid hormone. J Clin Endocrinol Metab 100: 2841-2848.

4. Moran C, Schoenmakers N, Agostini M, Schoenmakers E, Offiah A, Kydd A, Kahaly G, Mohr-Kahaly S, Rajanayagam O, Lyons G, Wareham N, Halsall D, Dattani M, Hughes S, Gurnell M, Park SM, Chatterjee K 2013 An adult female with resistance to thyroid hormone mediated by defective thyroid hormone receptor alpha. J Clin Endocrinol Metab 98:4254-4261.

5. Moran C, Agostini M, Visser WE, Schoenmakers E, Schoenmakers N, Offiah AC, Poole K, Rajanayagam O, Lyons G, Halsall D, Gurnell M, Chrysis D, Efthymiadou A, Buchanan C, Aylwin S, Chatterjee KK 2014 Resistance to thyroid hormone caused by a mutation in thyroid hormone receptor (TR)alpha1 and TRalpha2: clinical, biochemical, and genetic analyses of three related patients. Lancet Diabet Endocrinol 2:619-626.

6. Moran C, Agostini M, McGowan A, Schoenmakers E, Fairall L, Lyons G, Rajanayagam O, Watson L, Offiah A, Barton J, Price S, Schwabe J, Chatterjee K 2017 Contrasting phenotypes in resistance to thyroid hormone alpha correlate with divergent properties of thyroid hormone receptor alpha1 mutant proteins. Thyroid 27:973-982.

7. Tylki-Szymanska A, Acuna-Hidalgo R, KrajewskaWalasek M, Lecka-Ambroziak A, Steehouwer M, Gilissen C, Brunner HG, Jurecka A, Rozdzynska-Swiatkowska A, Hoischen A, Chrzanowska KH 2015 Thyroid hormone resistance syndrome due to mutations in the thyroid hormone receptor alpha gene (THRA). J Med Genet 52:312-316.

8. van Gucht AL, Meima ME, Zwaveling-Soonawala N, Visser WE, Fliers E, Wennink JM, Henny C, Visser TJ, Peeters RP, van Trotsenburg AS 2016 Resistance to thyroid hormone alpha in an 18-month-old girl: clinical, therapeutic, and molecular characteristics. Thyroid 26:338-346.

9. Demir K, van Gucht AL, Buyukinan M, Catli G, Ayhan Y, Bas VN, Dundar B, Ozkan B, Meima ME, Visser WE, Peeters RP, Visser TJ 2016 Diverse genotypes and phenotypes of three novel thyroid hormone receptor-alpha mutations. J Clin Endocrinol Metab 101:2945-2954.

10. van Gucht ALM, Moran C, Meima ME, Visser WE, Chatterjee K, Visser TJ, Peeters RP 2017 Resistance to thyroid hormone due to heterozygous mutations in thyroid hormone receptor alpha. Curr Top Dev Biol 125:337-355.

11. van Mullem A, van Heerebeek R, Chrysis D, Visser E, Medici M, Andrikoula M, Tsatsoulis A, Peeters R, Visser TJ 2012 Clinical phenotype and mutant TRalpha1. N Engl J Med 366:1451-1453.

12. van Mullem AA, Chrysis D, Eythimiadou A, Chroni E, Tsatsoulis A, de Rijke YB, Visser WE, Visser TJ, Peeters 
RP 2013 Clinical phenotype of a new type of thyroid hormone resistance caused by a mutation of the TRalpha1 receptor: consequences of LT4 treatment. J Clin Endocrinol Metab 98:3029-3038.

13. Astapova I, Hollenberg AN 2013 The in vivo role of nuclear receptor corepressors in thyroid hormone action. Biochim Biophys Acta 1830:3876-3881.

14. Vella KR, Ramadoss P, Costa ESRH, Astapova I, Ye FD, Holtz KA, Harris JC, Hollenberg AN 2014 Thyroid hormone signaling in vivo requires a balance between coactivators and corepressors. Mol Cell Biol 34:1564-1575.

15. Bassett JH, Boyde A, Zikmund T, Evans H, Croucher PI, Zhu X, Park JW, Cheng SY, Williams GR 2014 Thyroid hormone receptor alpha mutation causes a severe and thyroxine-resistant skeletal dysplasia in female mice. Endocrinology 155:3699-3712.

16. Kaneshige M, Kaneshige K, Zhu X, Dace A, Garrett L, Carter TA, Kazlauskaite R, Pankratz DG, Wynshaw-Boris A, Refetoff S, Weintraub B, Willingham MC, Barlow C, Cheng S 2000 Mice with a targeted mutation in the thyroid hormone beta receptor gene exhibit impaired growth and resistance to thyroid hormone. Proc Natl Acad Sci U S A 97:13209-13214.

17. Kaneshige M, Suzuki H, Kaneshige K, Cheng J, Wimbrow H, Barlow C, Willingham MC, Cheng S 2001 A targeted dominant negative mutation of the thyroid hormone alpha 1 receptor causes increased mortality, infertility, and dwarfism in mice. Proc Natl Acad Sci U S A 98:15095-15100.

18. Astapova I, Lee LJ, Morales C, Tauber S, Bilban M, Hollenberg AN 2008 The nuclear corepressor, NCoR, regulates thyroid hormone action in vivo. Proc Natl Acad Sci U S A 105:19544-19549.

19. Astapova I, Dordek MF, Hollenberg AN 2009 The thyroid hormone receptor recruits NCoR via widely spaced receptor-interacting domains. Mol Cell Endocrinol 307: 83-88.

20. Astapova I, Vella KR, Ramadoss P, Holtz KA, Rodwin BA, Liao XH, Weiss RE, Rosenberg MA, Rosenzweig A, Hollenberg AN 2011 The nuclear receptor corepressor (NCoR) controls thyroid hormone sensitivity and the set point of the hypothalamic-pituitary-thyroid axis. Mol Endocrinol 25: 212-224.

21. Fozzatti L, Kim DW, Park JW, Willingham MC, Hollenberg AN, Cheng SY 2013 Nuclear receptor corepressor (NCOR1) regulates in vivo actions of a mutated thyroid hormone receptor alpha. Proc Natl Acad Sci U S A 110: 7850-7855.

22. Kim DW, Park JW, Willingham MC, Cheng SY 2014 A histone deacetylase inhibitor improves hypothyroidism caused by a TRalpha1 mutant. Hum Mol Genet 23:26512664.

23. Bassett JH, Boyde A, Howell PG, Bassett RH, Galliford TM, Archanco M, Evans H, Lawson MA, Croucher P, St Germain DL, Galton VA, Williams GR 2010 Optimal bone strength and mineralization requires the type 2 iodothyronine deiodinase in osteoblasts. Proc Natl Acad Sci U S A 107:7604-7609.

24. Bassett JH, van der Spek A, Gogakos A, Williams GR 2012 Quantitative X-ray imaging of rodent bone by Faxitron. Methods Mol Biol 816:499-506.

25. Bassett JH, Logan JG, Boyde A, Cheung MS, Evans H, Croucher P, Sun XY, Xu S, Murata Y, Williams GR 2012 Mice lacking the calcineurin inhibitor Rcan2 have an iso- lated defect of osteoblast function. Endocrinology 153: 3537-3548.

26. Hones GS, Rakov H, Logan J, Liao XH, Werbenko E, Pollard AS, Praestholm SM, Siersbaek MS, Rijntjes E, Gassen J, Latteyer S, Engels K, Strucksberg KH, Kleinbongard P, Zwanziger D, Rozman J, Gailus-Durner V, Fuchs H, Hrabe de Angelis M, Klein-Hitpass L, Kohrle J, Armstrong DL, Grontved L, Bassett JHD, Williams GR, Refetoff S, Fuhrer D, Moeller LC 2017 Noncanonical thyroid hormone signaling mediates cardiometabolic effects in vivo. Proc Natl Acad Sci U S A 114:E11323e11332.

27. Schriefer JL, Robling AG, Warden SJ, Fournier AJ, Mason JJ, Turner CH 2005 A comparison of mechanical properties derived from multiple skeletal sites in mice. J Biomech 38: 467-475.

28. Moran C, Chatterjee K 2016 Resistance to thyroid hormone alpha-emerging definition of a disorder of thyroid hormone action. J Clin Endocrinol Metab 101:2636-2639.

29. Fozzatti L, Lu C, Kim DW, Park JW, Astapova I, Gavrilova O, Willingham MC, Hollenberg AN, Cheng SY 2011 Resistance to thyroid hormone is modulated in vivo by the nuclear receptor corepressor (NCOR1). Proc Natl Acad Sci U S A 108:17462-17467.

30. Shimizu H, Astapova I, Ye F, Bilban M, Cohen RN, Hollenberg AN $2015 \mathrm{NCoR} 1$ and SMRT play unique roles in thyroid hormone action in vivo. Mol Cell Biol 35:555-565.

31. Mendoza A, Astapova I, Shimizu H, Gallop MR, AlSowaimel L, MacGowan SMD, Bergmann T, Berg AH, Tenen DE, Jacobs C, Lyubetskaya A, Tsai L, Hollenberg AN 2017 NCoR1-independent mechanism plays a role in the action of the unliganded thyroid hormone receptor. Proc Natl Acad Sci U S A 114:E8458-E8467.

32. Richon VM 2006 Cancer biology: mechanism of antitumour action of vorinostat (suberoylanilide hydroxamic acid), a novel histone deacetylase inhibitor. Br J Cancer 95:S2-6.

33. Yeo P, Xin L, Goh E, New LS, Zeng P, Wu X, Venkatesh $P$, Kantharaj E 2007 Development and validation of highperformance liquid chromatography-tandem mass spectrometry assay for 6-(3-benzoyl-ureido)-hexanoic acid hydroxyamide, a novel HDAC inhibitor, in mouse plasma for pharmacokinetic studies. Biomed Chromatogr 21: 184-189.

34. O'Shea PJ, Bassett JH, Sriskantharajah S, Ying H, Cheng SY, Williams GR 2005 Contrasting skeletal phenotypes in mice with an identical mutation targeted to thyroid hormone receptor alpha1 or beta. Mol Endocrinol 19:30453059 .

Address correspondence to: J.H. Duncan Bassett, BMBCh, PhD, or Graham R. Williams, MBBS, PhD Molecular Endocrinology Laboratory Commonwealth Building, Hammersmith Campus Imperial College London Du Cane Road London W12 ONN United Kingdom

E-mail: d.bassett@imperial.ac.uk or graham.williams@imperial.ac.uk 